\title{
Genetic Learning of Membership Functions for Mining Fuzzy Association Rules
}

\author{
Rafael Alcalá and Jesús Alcalá-Fdez and M.J. Gacto and Francisco Herrera
}

\begin{abstract}
Data mining is most commonly used in attempts to induce association rules from transaction data. Most previous studies focused on binary-valued transaction data. Transaction data in real-world applications, however, usually consists of quantitative values. In the last years, the fuzzy set theory has been applied to data mining for finding interesting association rules in quantitative transactions.

Recently, a new rule representation model was presented to perform a genetic lateral tuning of membership functions. It is based on the 2-tuples linguistic representation model allowing us to adjust the context associated to the linguistic label membership functions.

Based on the 2-tuples linguistic representation model, we present a new fuzzy data-mining algorithm for extracting both association rules and membership functions by means of an evolutionary learning of the membership functions, using a basic method for mining fuzzy association rules.
\end{abstract}

\section{INTRODUCTION}

Data Mining (DM) is the process for automatic discovery of high level knowledge by obtaining information from real data. Some important problems that DM deal with are: rule extraction, identification of associations, feature analysis, linguistic summarization, clustering, classifier design and novelty/anomaly detection [1].

Association rules are used to represent and identify dependencies between attributes in a database [2]. Most previous studies focused on database with binary values [3], however the data in real-world applications usually consist of quantitative values. Designing sophisticated DM algorithms, able to deal with various types of data, presents a challenge to workers in this research field.

Lately, fuzzy set theory has been used more and more frequently in intelligent systems because of its simplicity and similarity to human reasoning [4]. The use of fuzzy sets to describe association between data extends the types of relationships that may be represented, facilitates the interpretation of rules in linguistic terms, and avoids unnatural boundaries in the partitioning of the attribute domains [5], [6].

Many researchers have proposed methods for mining fuzzy association rules from quantitative data [7], [8], [9], where the membership functions (MFs) were assumed to be known in advance. The given MFs may have a critical influence on

Rafael Alcalá, Francisco Herrera and M.J. Gacto are with the Department of Computer Science and Artificial Intelligence, University of Granada, Granada, 18071, Spain (phone: +34 958 244019; email: \{alcala, herrera\}@decsai.ugr.es, mjgacto@ugr.es).

Jesús Alcalá-Fdez is with the Department of Computer Science, University of Jaén, Jaén, 23071, Spain (phone: +34 953 212477; email: jalcala@decsai.ugr.es). the final mining results. For this reason, some approaches have also achieved a learning or tuning of the MFs [10], [11], [12], [13], [14].

Recently, a new linguistic rule representation model was proposed to perform a genetic lateral tuning of MFs [15]. This new approach was based on the 2-tuples linguistic representation [16], that allows the symbolic translation of a linguistic term by considering only one parameter per linguistic term. In this way, two main objectives were achieved:

- to obtain MFs with a more adequate context by maintaining a high covering degree of the data, and

- to reduce the search space respect to the classic learning [17], [18], [19] (usually considering three parameters in the case of triangular MFs), in order to easily obtain optimal models.

Based on the 2-tuples linguistic representation model, in this work, we present a new fuzzy data-mining algorithm for extracting both association rules and MFs from quantitative transactions by means of an evolutionary learning of the MFs and the use of the method presented in [8] for mining the fuzzy association rules. In this way, the search space reduction provided by the 2-tuples linguistic representation helps the evolutionary search technique to obtain more suitable MFs.

The paper is arranged as follows. The next section describes the linguistic rule representation model based on the linguistic 2-tuples. Section III introduces the mining scheme considered in this work. Section IV details the evolutionary learning algorithm proposed to obtain the MFs. Section V describes the proposed mining algorithm. Section VI shows the results of the proposed mining algorithm applied over a real-world problem. Finally, Section VII points out some conclusions.

\section{Preliminaries: The 2-tuPles linguistic REPRESENTATION}

The 2-tuples linguistic representation scheme presented in [16], introduces a new model for rule representation based on the concept of symbolic translation (the lateral displacement of a linguistic term).

The symbolic translation of a linguistic term is a number within the interval $[-0.5,0.5)$ that expresses the domain of a linguistic term when it is moving between its two lateral linguistic term. Let us consider a set of linguistic terms $S$ representing a fuzzy partition. Formally, we have the pair,

$$
\left(s_{i}, \alpha_{i}\right), s_{i} \in S, \alpha_{i} \in[-0.5,0.5) .
$$


Figure 1 depicts the symbolic translation of a linguistic term represented by the pair $\left(S_{2},-0.3\right)$, considering a set $S$ with five linguistic terms represented by their ordinal values $(\{0,1,2,3,4\})$.

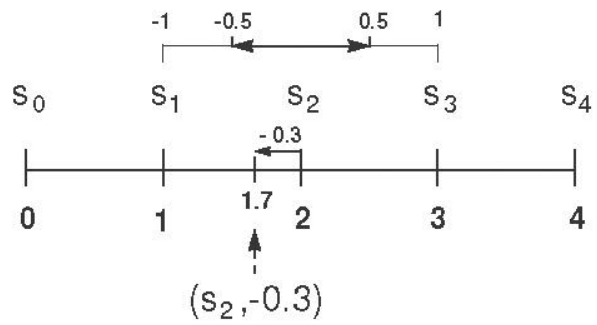

a) Simbolic Translation of a Linguistic Term

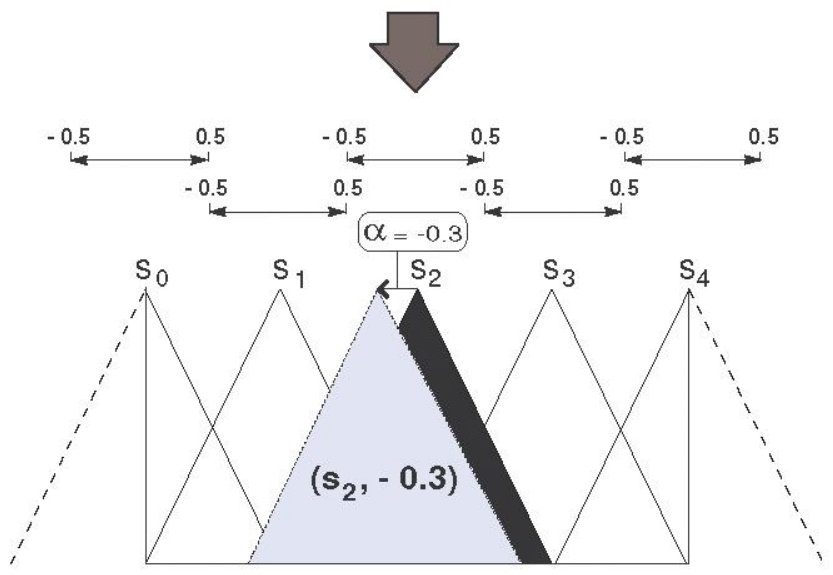

b) Lateral Displacement of a Membership Function

Fig. 1. Symbolic translation of a linguistic term and lateral displacement of the involved MF

In [16], both the 2-tuples linguistic representation model and the needed elements for linguistic information comparison and aggregation are presented and applied to the Decision Making framework. In [15], a new rule representation model has been presented based on these concepts to perform a tuning of complex linguistic fuzzy models. Now, we are going to extend its use for association rule representation. Below we present this approach considering a simple mining problem.

Let us consider a simple problem with two items (age and weight) and tree linguistic terms with their associated MFs (see Figure 2).
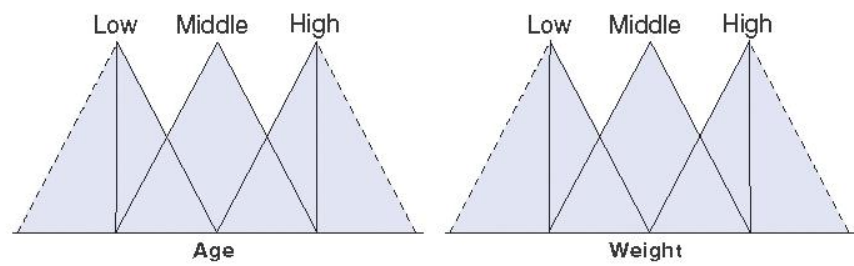

Fig. 2. Items and linguistic terms in a simple problem

Based on this definition, an example of classic association rule and 2-tuples linguistic represented rule is:
Classic Association Rule,

If the Age is Middle then the Weight is High.

\section{Rule with 2-Tuples Representation,}

If the Age is (Middle,0.3) then the Weight is (High,-0.1).

Analyzed from the rule interpretability point of view, we could interpret the 2-tuples linguistic represented rule in the following way:

If the Age is "higher than Middle"

then the Weight is "a bit smaller than High".

This proposal decreases the tuning problem complexity, since the three parameters usually considered per linguistic term [17], [18], [19] are reduced to only one symbolic translation parameter. Moreover, from the point of view of interpretability:

- the original shapes of the MFs are maintained (in our case triangular and symmetrical), by laterally changing the location of their supports,

- the lateral variation of the involved MFs is restricted to a short interval, ensuring overlapping between two adjacent $M F$ s to some degree but preventing their vertex points from crossing, and

- the 2-tuples represented linguistic terms can be interpreted with respect to the initial ones.

\section{The Mining Scheme}

An efficient way to generate the fuzzy association rules consists of learning the MFs a priori and then use the final best set of MFs to mine fuzzy association rules [11], [13], [14]. This way to work allows us to learn the most adequate context [20] for each fuzzy partition, which is necessary in different contextual situations (different applications).

The scheme considered for discovering both useful fuzzy association rules and suitable MFs from quantitative values is comprised of two main stages (see Figure 3):

1) An evolutionary process to learn the MFs.

2) A method to mine fuzzy association rules. The method presented in [8] will be considered for this task as a first approach.

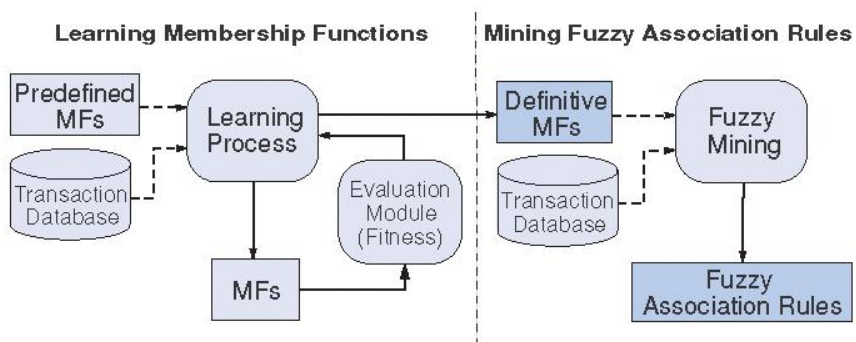

Fig. 3. Scheme for discovering both useful fuzzy association rules and suitable MFs 


\section{EVOLUTIONARY LEARNING ALGORITHM}

The automatic definition of fuzzy systems can be considered as an optimization or search process and nowadays Evolutionary Algorithms, particularly Genetic Algorithms (GAs), are considered as the more known and used global search technique. Moreover, the genetic coding that they use allow them to include prior knowledge and to use it leading the search up. For this reason, Evolutionary Algorithms have been successfully applied to learn and to tune fuzzy systems in the last years.

In this work, we will consider the use of GAs to design the proposed learning method of the MFs. A good evolution model is the CHC evolutionary model [21]. The CHC algorithm is a GA that presents a good trade-off between exploration and exploitation, being a good choice in problems with complex search spaces.

In the following, the components needed to design this process are explained. They are:

- CHC Evolutionary model.

- MFs codification and initial gene pool.

- Chromosome evaluation.

- Genetic operators.

\section{A. CHC Evolutionary Model}

We will consider a population-based selection approach, by using the $\mathrm{CHC}$ evolutionary model [21] in order to perform an adequate global search. The genetic model of CHC makes use of a "Population-based Selection" approach. $N$ parents and their corresponding offspring are combined to select the best $N$ individuals to take part of the next population. The CHC approach makes use of an incest prevention mechanism and a restarting process to provoke diversity in the population, instead of the well known mutation operator.

This incest prevention mechanism will be considered in order to apply the crossover operator, i.e., two parents are crossed if their hamming distance divided by 2 is over a predetermined threshold, $L_{T}$. Since, we will consider a real coding scheme, we have to transform each gene considering a Gray Code with a fixed number of bits per gene (BITSGENE) determined by the system expert. In this way, the threshold value is initialized as:

$$
L_{T}=(\# \text { Genes } * \text { BITSGENE) } / 4.0,
$$

where \#Genes is the number of genes in the chromosome. Following the original CHC scheme, $L_{T}$ is decremented by one when there is no new individuals in the population in one generation. In order to make this procedure independent of \#Genes and BITSGENE, in our case, $L_{T}$ will be decremented by a $\varphi \%$ of its initial value (being $\varphi$ determined by the user, usually $10 \%$ ). The algorithm restarts when $L_{T}$ is below zero.

A scheme of this algorithm is shown in Figure 4.

\section{B. MFs Codification and Initial Gene Pool}

A real coding scheme is considered, i.e., the real parameters are the GA representation units (genes). Each chromosome is a vector of real numbers with size $n * m$ ( $n$ items with

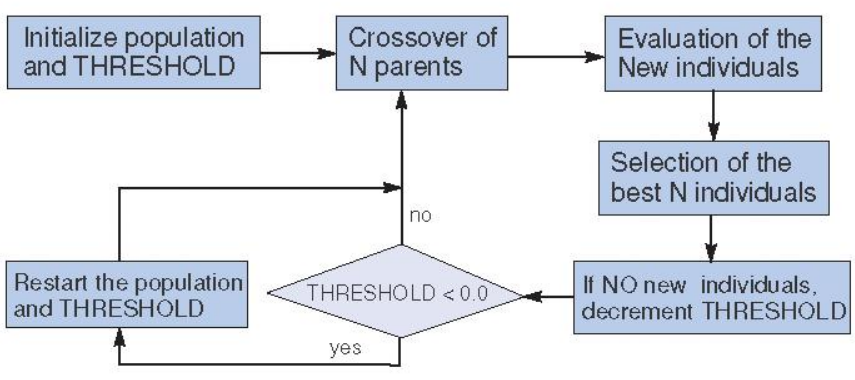

Fig. 4. Scheme of $\mathrm{CHC}$

$m$ linguistic terms per item) in which the displacements of the different linguistic terms are coded for each item. Then, a chromosome has the following form (where each gene is the tuning value of the corresponding linguistic term),

$$
\left(c_{11}, \ldots, c_{1 m}, c_{21}, \ldots, c_{2 m}, \ldots, c_{n 1}, \ldots, c_{n m}\right)
$$

Figure 5 graphically depicts an example of correspondence between a chromosome and its associated MFs. Notice that, the three parameters usually considered per linguistic term (in the case of triangular MFs) are reduced to only one parameter.

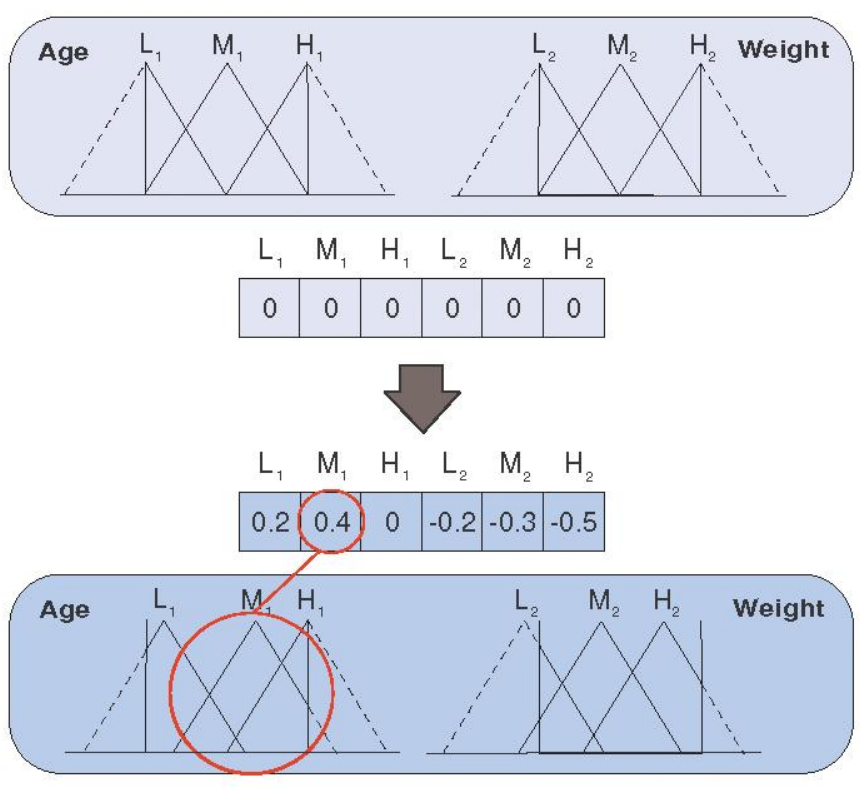

Fig. 5. Example of coding scheme

To make use of the available information, the initial MFs obtained from expert knowledge are included in the population as an initial solution. To do so, the initial pool is obtained with the first individual having all genes with value ' 0.0 ', and the remaining individuals generated at random in $[-0.5,0.5)$.

\section{Chromosome Evaluation}

To evaluate a determined chromosome we will use the fitness functions defined in [14]. Before the fitness of each set of MFs is formally described, several related terms are explained below. 
The overlap ratio of two MFs $R_{i}$ and $R_{j}(i<j)$ is defined as the overlap length divided by half the minimum span of the two functions. If the overlap length is larger than half the span, then these two MFs are thought of as a little redundant. Appropriate punishment must then be considered in this case. Thus, the overlap factor of the MFs for an item $I_{k}$ in the chromosome $C_{q}$ is defined as

$$
\sum_{i=1}^{m} \sum_{j=i+1}^{m}\left[\max \left(\frac{\operatorname{overlap}\left(R_{i}, R_{j}\right)}{\min \left(\operatorname{span} R_{i}, \operatorname{span} R_{j}\right)}, 1\right)-1\right]
$$

where overlap $\left(R_{i}, R_{j}\right)$ is the overlap length of $R_{i}$ and $R_{j}$, and $m$ is the number of MFs for $I_{k}$. Notice that the span of the two MFs is the same because in our case the original shapes of the MFs are maintained (triangular and symmetrical).

The coverage ratio of MFs for an item $I_{k}$ is defined as the coverage range of the functions divided by the maximum quantity of that item in the transactions. The more the coverage ratio is, the better the derived MFs are. Thus, the coverage factor of the MFs for an item $I_{k}$ in the chromosome $C_{q}$ is defined as:

$$
\frac{1}{\frac{\operatorname{range}\left(R_{1}, \ldots, R_{m}\right)}{\max \left(I_{k}\right)}}
$$

where range $\left(R_{1}, R_{2}, \ldots, R_{m}\right)$ is the coverage range of the MFs and $\max \left(I_{k}\right)$ is the maximum quantity of $I_{k}$ in the transactions. Notice that the coverage factor is always 1 because in our case the 2-tuples linguistic representation ensures the coverage in all the dominion, reducing the computation time. The suitability of the set of MFs in a chromosome $C_{q}$, is therefore defined as:

$$
\sum_{i=1}^{n}\left[\text { overlap_factor }\left(C_{q^{i}}\right)+1\right]
$$

where $n$ is number of items. The fitness value of a chromosome $C_{q}$ is then defined as

$$
\operatorname{fitness}\left(C_{q}\right)=\frac{\left|L_{1}\right|}{\text { suitability }\left(C_{q}\right)}
$$

where $\left|L_{1}\right|$ is the number of large 1-itemsets obtained by using the set of MFs in $C_{q}$. The suitability factor can reduce the occurrence of the two bad kinds of MFs shown in Figure 6, where the first one is too redundant, and the second one is too separate. The overlap factor in suitable $\left(C_{q}\right)$ is used for avoiding the first bad case, and the 2-tuples linguistic representation prevents the second one.
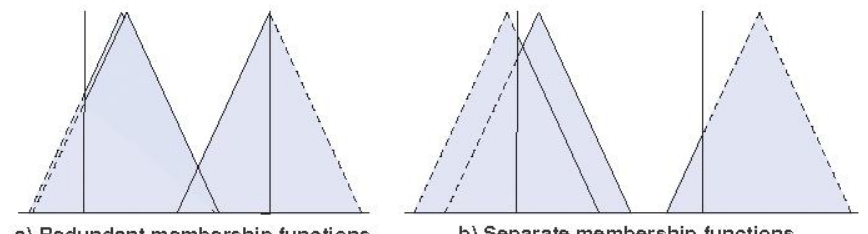

Fig. 6. Two bad kinds of membership functions

\section{Genetic Operator}

The genetic operators considered in $\mathrm{CHC}$ are crossover and restart approach (no mutation is considered). A description of these operators is presented in the following:

- Crossover. The crossover operator is based on the concept of neighborhood. These kinds of operators show a good behavior as is shown in [22]. Particularly, we consider the PBLX operator (an operator based on the BLX- $\alpha$ ). This operator presents a good cooperation when it is introduced within models forcing the convergence by pressure on the offspring. Figure 7 shows the performance of these kinds of operators, which allow the offspring genes to be around the genes of one parent (PCBLX) or around a wide zone determined by both parent genes (BLX).

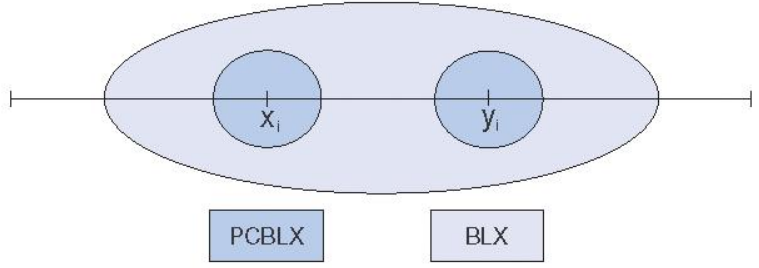

Fig. 7. Diagram of the performance of the crossover operators based on environments

The PCBLX is described as follows. Let us assume that $X=\left(x_{1} \cdots x_{n}\right)$ and $Y=\left(y_{1} \cdots y_{n}\right),\left(x_{i}, y_{i} \in\right.$ $\left.\left[a_{i}, b_{i}\right] \subset \Re, i=1 \cdots n\right)$, are two real-coded chromosomes that are going to be crossed. The PCBLX operator generates the two following offspring:

- $O_{1}=\left(o_{11} \cdots o_{1 n}\right)$, where $o_{1 i}$ is a randomly (uniformly) chosen number from the interval $\left[l_{i}^{1}, u_{i}^{1}\right]$, with $l_{i}^{1}=\max \left\{a_{i}, x_{i}-I_{i}\right\}, u_{i}^{1}=\min \left\{b_{i}, x_{i}+I_{i}\right\}$, and $I_{i}=\left|x_{i}-y_{i}\right|$.

- $O_{2}=\left(o_{21} \cdots o_{2 n}\right)$, where $o_{2 i}$ is a randomly (uniformly) chosen number from the interval $\left[l_{i}^{2}, u_{i}^{2}\right]$, with $l_{i}^{2}=\max \left\{a_{i}, y_{i}-I_{i}\right\}$ and $u_{i}^{2}=\min \left\{b_{i}, y_{i}+\right.$ $\left.I_{i}\right\}$.

- Restart approach. To get away from local optima, this algorithm uses a restart approach [21]. In this case, the best chromosome is maintained and the remaining are generated at random within the corresponding variation intervals $[-0.5,0.5)$. It follows the principles of $\mathrm{CHC}$ [21], performing the restart procedure when a threshold value is reached or all the individuals coexisting in the population are very similar.

\section{Proposed Mining Algorithm}

According to the above description, the proposed algorithm for mining both MFs and fuzzy association rules is described below.

INPUT: $T$ quantitative transaction data, a set of $n$ items, each with $m$ predefined linguistic terms, a support threshold $\alpha$, a confidence threshold $\lambda$ and a population size $N$. 
OUTPUT: A set of fuxxy association tules with its asociaded set of WIFs.

\section{Stage 1. Evoktionary learning of tha MFs.}

Step $1:$ : Geretate the iritiel population with $N$ chtomosonces. Step 2: Evaluade the population. For each chtromosonce:

- For each trabsetion datum $D_{b}$, $=1$ to $T$, and for each item $j, j=1$ to $n$, tansfer the quantitative value $\left.j_{j}\right)\left(D_{i}\right.$

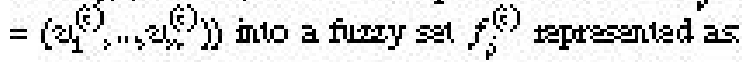

$$
f_{j}^{(0)}=\left(\frac{f_{j 1}^{(0)}}{R_{j 1}}+\ldots+\frac{f_{j \mathrm{r}}^{(\hat{\theta})}}{R_{j m}}\right)
$$

using the cotrefording MFs tepresented by the chtomosonce where $R_{\text {jis }}$ is the in -the linguistic tem of

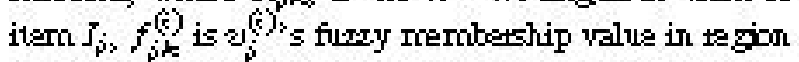
$R_{i b}$ and $m$ is the number of linguistic tems for $J_{j}$.

- For each linguistis term $R_{\text {ips }}$ caleulate is court on the tarkections as follows:

$$
\cos 2 t_{j k}=\sum_{i=1}^{T} f_{j i t}^{(i)}
$$

- For asch $R_{i+\infty} 1<j<n$ and $1<i<m$ ohock whe ther is cosot; latger than or equal to the minimoum support threshold $\alpha$. If $R_{\text {; t }}$ stisfies the above condition, put it in the set of bage 1-iternees $\left(L_{1}\right)$. That is:

$$
L_{1}=\left\{R_{j, k} \mid<00 \sec t_{j, k} \geq \alpha, 1 \leq j \leq n \text { osed } 1 \leq k \leq n\right\}
$$

- Set the fulves value of the chtormosome as the number of linguistic emo in $L_{1}$ divited by sestab aty $\left(C_{9}\right)$. That is:

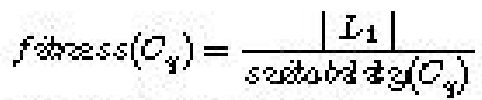

Steg 3: Iritialize the thas shold value $I$.

Step 4: Geretade the next population:

- Shuffle the population.

- Select the parents two by two. Each pair is ctosed (PCELX) if the hamrning ditance betwen the patent Gray coding divited by 2 is over $L$.

- Evaluate the new individuals.

- Joir the patents with their offepting and selset the best $N$ individuals to take pat of the rext population.

Step 5: If the best chtomosome does not change of there ate no new indivituals in the population, $L=L-\left(L_{\text {ixitios }}+0.1\right)$. Step 6 : If $L<0$, testart the poputation.

Step 7: If the maximom number of evabuations is not teached, go to Step 4.

Stage 2. Baric methad for ming fuzby association nules.

Step 8: The set of the best MIFs is then ued to mine furoy ascoiadion tules from the given quantitative database. The fuxoy mining algotithro propoest in [B] is then adopted to achieve this putpose.

\section{VI, EXPERDIENTAL REULTS}

To illustade the propoed mining algotithro a study of the athetcoshtosis is considered. It is a study of the tikk fectots of athetcocletcosis in a population of 1417 middle-aged men ${ }^{1}$. Hete, we extact five quantilative altibutes out of a total of 64. The selected attributes ate height weight systolis bloot ptessute, diestolic bloot ptessute, and cholesterl level

The values considered for the parameters of the proposed rethod are:

- Evolutionary ptocess: 50 individuak 10,000 evaluations and 0,6 as ctosoover probability for PBLX.

- Method for mining fuxyy assoiation rules: 0.7 for the confideruce thuesholi.

Figute 8 stows the telationship between the numbers of hate 1 -ilemeets and several values for the roirimoum suppott with one uriform fulugy partition and the poropoed methot. We can see that the leatring of the MIFs achieves latger a number of latze 1-itercests than the one with uriform fuxsy partitions consideting different values of minimom suppotl.

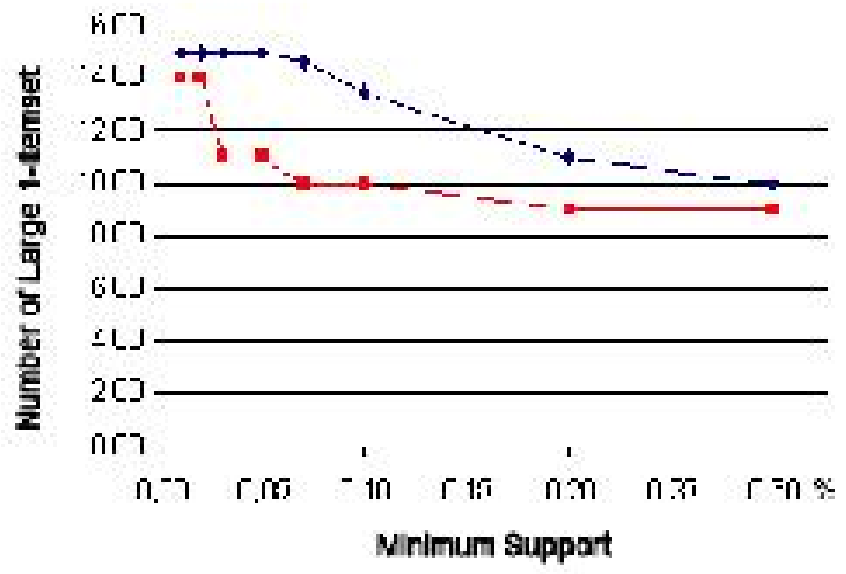

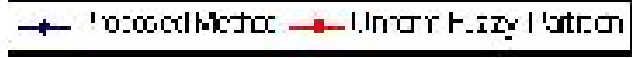

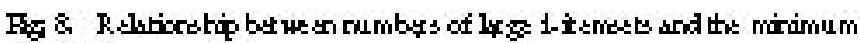
supports

Figute 9 depicts the MIFs obtained by the ptoposed method from one of the turs perfotred in this ptoblem with 3 linguistic terTos by attribute. This figute shons how snall displecernerts in the MIF las to inportant inctovernents in the number of obuined tules. The MFs ate mote or less well distributed which allows us to easily give a meaning to the conespouding linguistic terTcE.

One nule roined out with fertor of confidence 0.94 is shown as an exarqule:

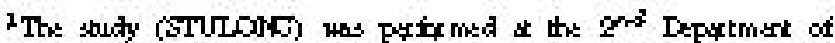

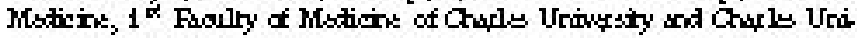
versity Hoppital undy the supquisian of Prof F Boud with oollsborsion

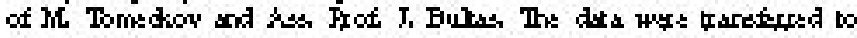

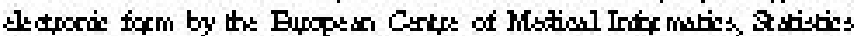

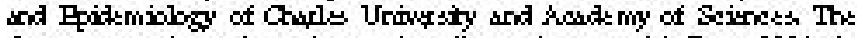

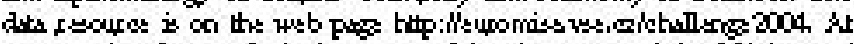

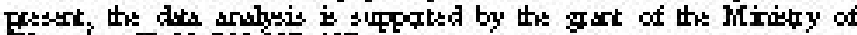
Ehuestion CR Jir LV TOE $10 \mathrm{~T}$ 

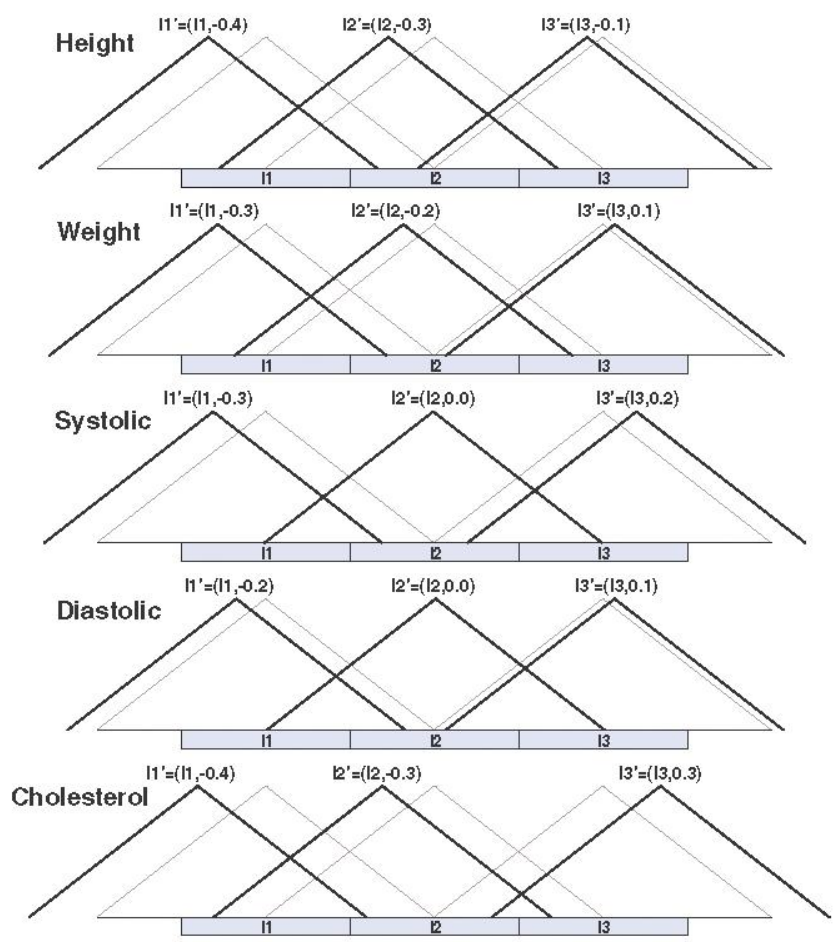

Fig. 9. MFs with/without lateral displacements (black/gray)

If the systolic blood pressure is (Low,-0.12) and the diastolic blood is (Low,-0.05) then the cholesterol level is (Middle,0.03)

\section{CONCLUSIONS}

In this work, a new rule representation scheme by using the 2-tuples linguistic representation model has been considered to extracting both MFs and fuzzy association rules from quantitative transactions by means of an evolutionary learning of the MFs and a later basic method to mine fuzzy association rules. Here, we present our conclusions and further considerations:

- The 2-tuples linguistic representation model allows an important reduction of the search space from the optimization point of view.

- The used learning scheme together with the 2-tuples linguistic representation model and the used fitness function offers a good mechanism to obtain interpretable association rules, since the shapes of the initial MFs are preserved, the MFs are maintained symmetrical and more or less well distributed, ensuring full coverage and the bad kinds of MFs are avoided in the mining process.

In the future, we will attempt to enhance the proposed method for more complex problems.

\section{ACKNOWLEDGMENT}

Supported by the Spanish Ministry of Science and Technology under Project TIN2005-08386-C05-01.

\section{REFERENCES}

[1] J. Han and M. Kamber, Data Mining: Concepts and Techniques, Second Edition, Morgan Kaufmann, 2006.

[2] Ch. Zhang and S. Zhang, Association Rule Mining: Models and Algorithms, Lecture Notes in Computer Science, LNAI 2307, 2002.

[3] R. Agrawal, T. Imielinski and A. Swami. "Mining association rules between sets of items in large databases", SIGMOD, Washington D.C., May 1993, pp. 207-216.

[4] H. Ishibuchi, T. Nakashima and M. Nii, Classification and Modeling with Linguistic Information Granules: Advanced Approaches to Linguistic Data Mining, Springer, Berlin, 2004.

[5] M. Delgado, N. Maŕn, D. Sánchez and M.-A. Vila, "Fuzzy association rules: General model and applications", IEEE Transactions on Fuzzy Systems, vol. 11, no. 2, pp. 214-225, 2003.

[6] D. Dubois, H. Prade and T. Sudamp, "On the representation, measurement, and discovery of fuzzy associations", IEEE Transactions on Fuzzy Systems, vol. 13, pp. 250-262, 2005.

[7] S. Yue, E. Tsang, D. Yeung and D. Shi, "Mining fuzzy association rules with weighted items", IEEE International Conference on Systems, Man and Cybernetics, Nashville, Tennessee, October 2000, pp. 1906-1911.

[8] T.P. Hong, C.S. Kuo and S.C. Chi, "Trade-off between time complexity and number of rules for fuzzy mining from quantitative data", International Journal Uncertain Fuzziness Knowledge-Based Systems, vol. 9, no. 5, pp. 587-604, 2001.

[9] Y.C. Lee, T.P. Hong and W.Y. Lin, "Mining fuzzy association rules with multiple minimum supports using maximum constraints", Lecture Notes in Computer Science, vol. 3214, pp. 1283-1290, 2004.

[10] W. Wang and S.M. Bridges, "Genetic Algorithm Optimization of Membership Functions for Mining Fuzzy Association Rules", International Joint Conference on Information Systems, Fuzzy Theory and Technology Conference, Atlantic City, N.Y., March 2000, pp. 1-4.

[11] M. Kaya and R. Alhajj, "A clustering algorithm with genetically optimized membership functions for fuzzy association rules mining", IEEE International Conference on Fuzzy Systems, St. Louis, Missouri, 2003, pp. 881-886.

[12] M. Kaya and R. Alhajj, "Integrating multi-objective genetic algorithms into clustering for fuzzy association rules mining", Fourth IEEE International Conference on Data Mining, Brighton, UK, November 2004, pp. 431-434.

[13] T.P. Hong, C.H. Chen, Y.L. Wu and Y.C. Lee, "Using Divide-andConquer GA Strategy in Fuzzy Data Mining", Proc. IEEE International Symposium on Fuzzy Systems, Budapest, Hungary, July 2004, pp. 116121.

[14] T.P. Hong, C.H. Chen, Y.L. Wu and Y.C. Lee, "A GA-based fuzzy mining approach to achieve a trade-off between number of rules and suitability of membership functions", Soft Computing, vol. 10, pp. 1091$1101,2006$.

[15] R. Alcalá, J. Alcalá-Fdez and F. Herrera, "A Proposal for the Genetic Lateral Tuning of Linguistic Fuzzy Systems and its Interaction with Rule Selection", IEEE Transactions on Fuzzy Systems, (2007) In press.

[16] F. Herrera and L. Martíez, "A 2-tuple fuzzy linguistic representation model for computing with words", IEEE Transactions on Fuzzy Systems, vol. 8, no. 6, pp. 746-752, 2000.

[17] R. Babuska, J. Oosterhoff, A. Oudshoorn and P. M. Bruijn, "Fuzzy self-tuning PI control of $\mathrm{pH}$ in fermentation", Engineering Applications of Artificial Intelligence, vol. 15, no. 1, pp. 463-484, 2002.

[18] O. Cordon, F. Herrera, F. Hoffmann and L. Magdalena, GENETIC FUZZY SYSTEMS. Evolutionary tuning and learning of fuzzy knowledge bases. Advances in Fuzzy Systems - Applications and Theory, World Scientific, vol. 19, 2001.

[19] C. Karr, "Genetic algorithms for fuzzy controllers", AI Expert, vol. 6, no. 2, pp. 26-33, 1991.

[20] O. Cordón, F. Herrera, L. Magdalena and P. Villar, "A genetic learning process for the scaling factors, granularity and contexts of the fuzzy rule-based system data base", Information Sciences, vol. 136, pp. 85$107,2001$.

[21] L.J. Eshelman, "The CHC adaptive search algorithm: How to have safe search when engaging in nontraditional genetic recombination", in Foundations of genetic Algorithms, G. Rawlin, Ed. Morgan Kaufman, 1991, vol. 1, pp. 265-283.

[22] F. Herrera, M. Lozano and A.M. Sánchez, "A taxonomy for the crossover operator for real-coded genetic algorithms: An experimental study", International Journal of Intelligent Systems, vol. 18, pp. 309$338,2003$. 